\title{
Sistema de cultivo e doses de nitrogênio na sanidade e no rendimento do milho
}

\author{
Cropping systens and nitrogen levels in the maize plant health and yield
}

\author{
João Américo Wordell Filho ${ }^{*}$ Evandro Spagnollo $^{\mathrm{I}}$
}

\section{RESUMO}

\begin{abstract}
As podridões da base do colmo $(P B C)$ e grãos ardidos (GA) ocorrem em lavouras de milho, causando prejuízos aos produtores e contaminando os grãos com micotoxinas. $O$ objetivo deste estudo foi quantificar os efeitos do monocultivo de milho, da rotação de culturas com feijoeiro e da adubação nitrogenada na incidência de PBC e GA, nos teores de fumonisinas, peso de mil sementes (PMS) e no rendimento de grãos de milho. $O$ delineamento experimental adotado foi em blocos casualizados com parcelas subdivididas e quatro repetições por tratamento. Na parcela principal, foram testados três híbridos de milho: P30F36, AS 1575 e Maximus. Nas subparcelas, foi avaliada a resposta dos híbridos a cinco doses de nitrogênio $(N)$, equivalentes a 0; 75; 140; 215 e $290 \mathrm{~kg}$ de $N$ $h a^{-1}$, em ambos os sistemas de cultivo. Não foram observadas diferenças significativas para os valores médios de PBC, fumonisina e rendimento de grãos nos dois sistemas de cultivo avaliados. Para os valores médios de GA e PMS, ocorreram diferenças entre híbridos somente quando cultivados em rotação de cultura. A incidência dos parâmetros PBC e GA, quando em monocultura, foi superior aos encontrados quando cultivado em rotação de culturas. As variações médias do PMS e rendimento de grãos foram superiores quando em rotação, comparativamente ao sistema de cultivo em monocultura. As diferentes doses de $N$ explicaram apenas parcialmente as variações observadas em todas as variáveis analisadas.
\end{abstract}

Palavras-chave: micotoxinas, adubação nitrogenada, rotação de culturas, monocultura.

\section{ABSTRACT}

The stalk rot (SR) and grain rot $(G R)$ occur in crops as corn, causing losses to farmers and contaminating the grain with mycotoxins. The aim of this study was to quantify the effects of corn monoculture, crop rotation with common bean, and nitrogen fertilization on the incidence of $S R, G R$, fumonisin levels, weight of thousand seeds (WTS) and grain yield. The experiment was randomized blocks with split plot and four replicates per treatment. In the main plot were tested three maize hybrids: P30F36, AS 1575 and Maximus. In the subplots, each of the hybrids was evaluated in five levels of nitrogen $(N)$, equivalent to 0; 75; 140; 215 and 290kg of $N \mathrm{ha}^{-1}$ in both cropping systems. There were no significant differences for mean values of SR, Fumonisin and grain yield in both cropping systems evaluated. For GR and WTS average values had differences between hybrids only when corn was grown in crop rotation with common bean. The annual growth rate of SR and GR parameters in corn monoculture ranged from 44.8 to $172.4 \%$, above the rate found on corn when grown in crop rotation. In turn, mean changes of WTS and grain yield was 1.2 to $17.6 \%$ higher when in rotation compared to monoculture cropping system. The differences found in nitrogen rates partly explained the changes observed in all variables.

Key words: mycotoxins, nitrogen fertilization, crop rotation, monoculture.

\section{INTRODUÇÃO}

A cultura do milho (Zea mays L.) apresenta grande importância socioeconômica no Estado de Santa Catarina devido ao consumo desse cereal pelas cadeias produtivas das agroindústrias de carne e leite. Nesse Estado, anualmente são produzidas mais de 3,5 milhões de t de milho, porém com déficit superior a 1,5 milhão de $\mathrm{t}_{\text {ano }}{ }^{-1}$ (CEPA, 2011). A diferença entre a produção e o consumo pode ser minimizada e até anulada pela aplicação de adequadas práticas de manejo.

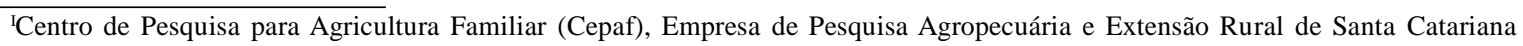
(Epagri), CP 791, 89801-970, Chapecó, SC, Brasil. E-mail: wordell@epagri.sc.gov.br. *Autor para correspondência. 
Santa Catarina possui uma estrutura fundiária predominantemente de pequenas propriedades rurais, com utilização intensiva do solo. Por isso, a rotação de culturas nem sempre é possível, favorecendo os patógenos que sobrevivem em restos culturais presentes na superfície do solo (CASA et al., 2003; WORDELLFILHO et al., 2008). Assim, aprimorar o manejo dos agentes bióticos consiste em alternativa para aumentar a produtividade das lavouras.

Dentre esses patógenos que danificam o milho, destacam-se os fungos que causam as podridões da base do colmo (PBC), pois reduzem a resistência das plantas. Com a base dos colmos afetada, a translocação de água e de nutrientes do solo para a parte aérea das plantas fica comprometida, reduzindo o rendimento da cultura e a qualidade dos grãos (SHURTLEFF, 1992).

As principais espécies de fungos causadoras de PBC relatadas no Brasil são: Colletotrichum graminicola (Ces), Stenocarpella maydis (Berk.) [Sin. Diplodia maydis (Berk.), Stenocarpella macrospora (Earle) [Sin. D. macrospora Earle in Bull.], Fusarium graminearum Schwabe (Gibberella zeae Schw.) e Fusarium verticillioides $[$ Sin. $=$ Fusarium moniliforme J. Sheld (Gibberella fujikuroi Sawada)] (WORDELL FILHO \& CASA, 2010). Muitos desses fungos estão também associados à ocorrência de grãos ardidos (GA), produzidos principalmente pela colonização da espiga durante a fase de enchimento de grãos. Esses GAs constituem um dos principais problemas de redução da qualidade de grãos de milho devido à produção de micotoxinas, incluindo as fumonisinas causadas pelos fungos $\boldsymbol{F}$. moniliforme, $\boldsymbol{F}$. verticillioides e $\boldsymbol{F}$. proliferatum. Essas micotoxinas provocam edema pulmonar e carcinomas hepáticos em suínos e aves, sendo a fumonisina a principal toxina encontrada no milho produzido no Oeste do Estado de Santa Catarina (WORDELLFILHO, 2010).

Neste trabalho, foi formulada a hipótese de que a incidência das $\mathrm{PBC}$ e os teores de fumonisinas em grãos de milho são influenciados pela presença e tipo de restos culturais na superfície do solo das lavouras e pelas doses de adubação nitrogenada utilizadas no cultivo do milho. Assim, o objetivo deste estudo foi quantificar os efeitos da monocultura do milho, da rotação de culturas e de doses de adubação nitrogenada na incidência de $\mathrm{PBC}$, rendimento de grãos, porcentagem de GA e teores de fumonisinas, além de identificar as principais espécies de fungos causadores de PBC nos dois sistemas de cultivo.

\section{MATERIAL E MÉTODOS}

O estudo foi desenvolvido no município de Chapecó, situado no Oeste do Estado de Santa Catarina, em uma área localizada a $27^{\circ} 06^{\prime} 34^{\prime \prime}$ de latitude Sul e $52^{\circ} 40^{\prime} 18^{\prime \prime}$ de longitude Oeste, com $623 \mathrm{~m}$ de altitude. $\mathrm{O}$ delineamento experimental foi em blocos casualizados com parcelas subdivididas e quatro repetições por tratamento. $\mathrm{O}$ espaçamento usado foi de $0,80 \mathrm{~m}$ entre filas, com população de 60.000 plantas $\mathrm{ha}^{-1}$. Na parcela principal $\left(288 \mathrm{~m}^{2}\right)$, foram testados três híbridos simples de milho de ciclo precoce: P30F36 (moderadamente resistente às PBC e moderadamente suscetível à GA) (PORTAL PIONEER, 2008), AS 1575 (tolerante às PBC e GA) e Maximus (moderadamente resistente às $\mathrm{PBC}$ e moderadamente suscetível à GA). Nas subparcelas $\left(96 \mathrm{~m}^{2}\right)$, foram avaliadas cinco doses de nitrogênio, equivalentes a 0; 75; 140; 215 e 290kg de $\mathrm{N} \mathrm{ha}^{-1}$, utilizando-se a uréia como fonte nitrogenada. As unidades experimentais consistiram de seis linhas de $5 \mathrm{~m}$ de comprimento.

Os ensaios foram conduzidos nas safras agrícolas de 2008/09 e 2009/10, em área cultivada no sistema de semeadura direta, com quatro anos de monocultivo de milho e, em área de semeadura de milho submetida a dois anos de rotação de culturas alternada com a cultura do feijão (Phaseolus vulgaris L.) Em ambas as áreas, o milho foi semeado em sucessão à cobertura morta de centeio (Secale cereale L.), dessecada com o herbicida glifosato, na dose de $1.400 \mathrm{ml}$ i.a. ha ${ }^{-1}$. A adubação com fósforo e potássio foi realizada de acordo com a análise de solo, para uma expectativa de rendimento de $9.600 \mathrm{~kg} \mathrm{ha}^{-1}$, seguindo as recomendações da Comissão de Química e Fertilidade do Solo (CQFS RS/SC, 2004), utilizando-se como fonte de nutrientes a fórmula NPK 8-20-20, super fosfato triplo (SFT) e cloreto de potássio $(\mathrm{KCl})$. A adubação nitrogenada de cobertura foi dividida em duas aplicações, realizadas aos 25 e 40 dias após a emergência (DAE).

A semeadura do milho foi realizada manualmente, em 23/09/2008 e 25/09/2009, 30 dias depois da dessecação da cobertura de inverno. Como prevenção ao ataque de pragas na fase de emergência das plântulas, as sementes foram tratadas com o inseticida tiametoxan (40g i.a por 100kg de sementes). Imediatamente após a semeadura, foi aplicada uma mistura de atrazina $\left(1.400 \mathrm{ml}\right.$ i.a. ha $\left.{ }^{-1}\right)$ e metolachlor $(2.100 \mathrm{ml} \mathrm{i.a.} \mathrm{ha-1)}$ sobre a superfície do solo para controlar plantas daninhas na pré-emergência do milho. A densidade final de plantas para a população préestabelecida foi obtida por desbaste manual, quando as plantas apresentaram três folhas expandidas. Para o 
controle da lagarta-do-cartucho, Spodoptera frugiperda (Smith) (Lepidoptera: Noctuidae), foi aplicada a mistura dos inseticidas diflubenzuron (100 $\left.\mathrm{g} \mathrm{ha}^{-1}\right)$ e metomil $\left(129 \mathrm{~g} \mathrm{ha}^{-1}\right)$ aos 45 dias após a emergência.

A incidência das $\mathrm{PBC}$ foi quantificada seguindo a metodologia proposta por REIS et al. (1998), considerando plantas sintomáticas aquelas que apresentaram descoloração do primeiro ou do segundo entrenó e/ou aquelas com menor resistência do colmo à pressão dos dedos polegar e indicador. Os colmos sintomáticos foram cortados e levados ao Laboratório de Fitossanidade da Epagri/Cepaf (Centro de Pesquisa para Agricultura Familiar), onde foram retirados dois fragmentos de tecido internos da medula de cada colmo da região próxima ao nó. Os fragmentos foram desinfetados com hipoclorito de sódio a $1 \%$, durante três minutos, e transferidos para caixas do tipo gerbox, contendo meio de cultura BDA (batata-dextrose-ágar = Merck: $\left.40 \mathrm{~g} \mathrm{~L}^{-1}\right)+\mathrm{A}\left(\right.$ antibiótico $=200 \mathrm{mg} \mathrm{L}^{-1}$ de sulfato de estreptomicina). Os dados obtidos foram expressos pela percentagem de incidência de $\mathrm{PBC}$, especificando os fungos associados.

Após a colheita, as espigas foram despalhadas e debulhadas manualmente, e os grãos foram secados em estufa até umidade padrão de $13 \%$, quantificando o peso de mil sementes (PMS) e o rendimento de grãos. A avaliação da percentagem de GA foi obtida pela separação manual de grãos sintomáticos e grãos sadios, em uma amostra de $250 \mathrm{~g}$ de cada parcela (BRASIL, 1996). Os GA foram pesados e a incidência indicada em percentagem. Também foi verificada a incidência de fungos nos grãos, utilizandose quatro repetições aleatórias de 200 grãos por tratamento, os quais foram desinfetados e transferidos para caixas gerbox em meio BDA+Antibiótico. O material foi incubado durante sete dias na temperatura de $25^{\circ} \mathrm{C}$ e fotoperíodo de $12 \mathrm{~h}$. Foram considerados infectados os grãos que desenvolveram colônias e/ou estruturas de fungos, os quais foram triados em microscópio estereoscópico, seguindo a metodologia adotada por CASA et al. (1998) e PINTO (1998). A quantificação de fumonisinas nas amostras de milho foi realizada de acordo com o plano amostral desenvolvido por FONSECA (1991) e metodologia de análise descrita por FARIAS et al. (2002).

Os dados de percentagem foram transformados em arco seno da raiz quadrada de $\mathrm{x} / 100$, submetidos à análise estatística pelo teste $\mathrm{F}$ e as médias comparadas pelo Teste de Tukey a 5\% de probabilidade, utilizando o programa estatístico Sanest (ZONTA \& MACHADO, 1987). Para os efeitos de doses de nitrogênio nas diferentes variáveis avaliadas, foram ajustadas equações de regressão.

\section{RESULTADOS E DISCUSSÃO}

Para as PBC, não foram observadas diferenças significativas na média dos dois anos de avaliação, no monocultivo de milho e na rotação com a cultura do feijão, porém foi observado efeito quando as análises estatísticas foram realizadas separadamente por ano (Tabela 1). Assim, no período de novembro de 2008 a janeiro de 2009, quando a precipitação pluviométrica foi $135,6 \mathrm{~mm}$ inferior do que no ano seguinte $(476,3 \mathrm{~mm})$, aconteceu menor incidência de PBC. A maior diferença pluviométrica ocorreu no mês de dezembro de 2008 , quando o volume de chuva foi 54\% inferior ao verificado em dezembro de 2009 (Estação Meteorológica do Centro de Pesquisa para Agricultura Familiar - Epagri).

Na safra 2008/2009, quando houve menor volume de chuvas, a intensidade de $\mathrm{PBC}$ seguiu a seguinte ordem: P30F36>Maximus>AS1575. Na safra seguinte (2009/2010), a ordem de intensidade de PBC foi: Maximus $>$ AS1575>P30F36, ou seja, os híbridos comportaram-se distintamente quando ocorreram diferentes condições climáticas. Além disso, as PBC foram influenciadas pelas doses de nitrogênio aplicadas na área de monocultura e nos períodos de menor pluviosidade (safra 2009/2010) (Figura 1). A incidência média de PBC na área de monocultura foi de 45,0 a $84,2 \%$ superior à encontrada no sistema de cultivo em rotação de culturas. Assim, mesmo nas menores doses de nitrogênio, os fatores que provavelmente determinaram o aumento das PBC devem estar relacionados com: competição por nutrientes, relação N e K (FOLEY \& WERNHAM, 1957), desequilíbrio nutricional entre os colmos e as espigas, disponibilidade de água, conforme sugerido por ZUBER et al. (1957) e SHURTLEFF (1992) e devido à nebulosidade prolongada (FANCELLI, 1994). Esses fatores são responsáveis pela rápida e prematura manifestação de senescência nos tecidos, favorecendo o aumento da suscetibilidade ao ataque de fungos causadores de PBC (DEACON, 1997).

DENTI \& REIS (2001), estudando o efeito da rotação de culturas, da monocultura e da densidade de plantas na incidência das $\mathrm{PBC}$, concluíram que a prática da monocultura aumenta a incidência de $\mathrm{PBC}$ na cultura do milho. A presença de restos culturais infectados assegura a sobrevivência dos patógenos nas lavouras, fato que contribui para a maior incidência dessas doenças na monocultura. Deve-se ressaltar que, na área de monocultura, no momento da semeadura em ambas as safras, foram quantificados restos culturais de milho em superfície na ordem de 2050 a 2200 $\mathrm{kg} \mathrm{ha}^{-1}$, remanescentes da safra anterior. Assim, as PBC do 
Tabela 1 - Variação média dos sistemas de cultivo nas podridões da base do colmo (\%), grãos ardidos (\%), Fusarium verticillioides (\%), peso de mil sementes (g) e rendimento de grãos $\left(\mathrm{kg} \mathrm{ha}^{-1}\right)$ avaliados na cultura do milho em dois sistemas de cultivo, nas safras 2008/2009 e 2009/2010. Chapecó, SC, 2011.

\begin{tabular}{|c|c|c|c|c|c|c|c|c|}
\hline \multirow{2}{*}{ Safra } & \multirow{2}{*}{ H $1 *$} & \multirow[b]{2}{*}{ H $2^{*}$} & \multirow[b]{2}{*}{ H $3 *$} & \multirow[b]{2}{*}{$\mathrm{CV}(\%)$} & \multirow[b]{2}{*}{ H 1} & \multirow{2}{*}{\multicolumn{2}{|c|}{$\begin{array}{l}\text { H } 2 \text {--Rotação de culturas--- } \\
\text { H } 3\end{array}$}} & \multirow[b]{2}{*}{$\mathrm{CV}(\%)$} \\
\hline & & & & & & & & \\
\hline \multicolumn{9}{|c|}{ 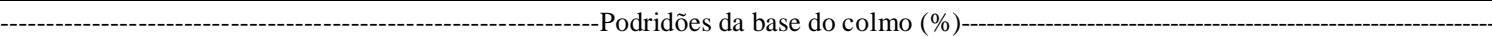 } \\
\hline 08/09 & $25,7 \mathrm{a}^{*}$ & $20,0 \mathrm{ab}$ & $14,2 \mathrm{~b}$ & 27,6 & $16,0 \mathrm{a}$ & $3,9 \mathrm{~b}$ & $3,1 \mathrm{~b}$ & 25,1 \\
\hline $09 / 10$ & $26,6 \mathrm{~ns}^{*}$ & 32,3 & 30,1 & 23,2 & $18,6 \mathrm{~b}$ & 32,2 a & $20,9 \mathrm{~b}$ & 21,4 \\
\hline Média & $26,2 \mathrm{~ns}$ & 26,1 & 22,1 & 42,8 & $17,3 \mathrm{~ns}$ & 18,0 & 12,0 & 42,8 \\
\hline$\Delta X^{*}$ & - & - & - & - & 51,5 & 45,0 & 84,2 & - \\
\hline & & & -----------F & mento de & $\left(\mathrm{kg} \mathrm{ha}^{-1}\right)$ & & & \\
\hline 08/09 & 7380,0 a & $5600,8 \mathrm{~b}$ & $5043,1 \mathrm{~b}$ & 16,1 & 8047,8 a & $6105,5 \mathrm{~b}$ & $6513,1 \mathrm{~b}$ & 20,4 \\
\hline $09 / 10$ & $9713,6 \mathrm{~ns}$ & 9219,8 & 9329,2 & 11,7 & $11150,6 \mathrm{a}$ & $10102,9 \mathrm{~b}$ & $10932,6 \mathrm{a}$ & 11,9 \\
\hline Média & $8546,8 \mathrm{~ns}$ & 7410,3 & 7186,1 & 30,2 & $9599,2 \mathrm{~ns}$ & 8104,2 & 8722,8 & 28,3 \\
\hline$\Delta \mathrm{X}$ & - & - & - & - & $-11,0$ & $-8,6$ & $-17,6$ & - \\
\hline 08/09 & $256,2 \mathrm{~b}$ & 288,5 a & 285,4 a & $\begin{array}{l}\text { de mil st } \\
8,1\end{array}$ & $\begin{array}{l}\text { tes }(\mathrm{g}) \\
274,9 \mathrm{~b}\end{array}$ & $292,9 \mathrm{ab}$ & 311,4 a & 9,9 \\
\hline $09 / 10$ & $322,4 \mathrm{~b}$ & $322,4 \mathrm{~b}$ & 356,9 a & 5,2 & $336,2 \mathrm{~b}$ & $325,1 \mathrm{c}$ & 374,7 a & 4,4 \\
\hline Média & $289,3 \mathrm{~ns}$ & 305,4 & 321,1 & 11,8 & $305,6 \mathrm{~b}$ & $309,0 \mathrm{~b}$ & $343,0 \mathrm{a}$ & 10,6 \\
\hline$\Delta \mathrm{X}$ & - & - & - & - & $-5,3$ & $-1,2$ & $-6,4$ & - \\
\hline 08/09 & $39,9 \mathrm{~b}$ & 58,8 a & $40,3 \mathrm{~b}$ & $\begin{array}{l}\text { Grãos a } \\
6,9\end{array}$ & $\begin{array}{r}\%(0,9 \mathrm{~b} \\
10\end{array}$ & $19,7 \mathrm{a}$ & $10,9 \mathrm{~b}$ & 20,4 \\
\hline $09 / 10$ & $9,0 \mathrm{c}$ & $24,0 \mathrm{a}$ & $16,0 \mathrm{~b}$ & 19,4 & $7,1 \mathrm{~b}$ & $16,7 \mathrm{a}$ & $14,3 \mathrm{a}$ & 20,4 \\
\hline Média & $24,4 \mathrm{~ns}$ & 41,4 & 28,2 & 30,0 & $9,0 \mathrm{c}$ & $18,2 \mathrm{a}$ & $12,6 \mathrm{~b}$ & 12,9 \\
\hline$\Delta \mathrm{X}$ & - & - & - & - & 171,1 & 127,5 & 123,8 & - \\
\hline 08/09 & $35,9 \mathrm{~b}$ & $41,2 \mathrm{~b}$ & $47,9 \mathrm{a}$ & $\begin{array}{l}\text { ium verti } \\
11,1\end{array}$ & $\begin{array}{c}\text { les }(\%) \\
36,0 \mathrm{~ns}\end{array}$ & 39,3 & 41,8 & 13,7 \\
\hline $09 / 10$ & $16,9 \mathrm{c}$ & $29,0 \mathrm{~b}$ & $34,5 \mathrm{a}$ & 13,3 & $18,6 \mathrm{c}$ & $29,4 \mathrm{~b}$ & $40,7 \mathrm{a}$ & 11,2 \\
\hline Média & $26,4 \mathrm{~b}$ & $35,1 \mathrm{ab}$ & $41,2 \mathrm{a}$ & 14,9 & $27,3 \mathrm{~b}$ & $34,3 \mathrm{ab}$ & 41,3 a & 11,8 \\
\hline$\Delta \mathrm{X}$ & - & - & - & - & $-3,3$ & 2,3 & $-0,2$ & - \\
\hline
\end{tabular}

*H1: P30F36, H2: Maximus, H3: AS1575; $\Delta \mathrm{X}$ : variação média do sistema de cultivo em rotação de culturas relativo ao sistema de cultivo em monocultura; ns: não significativo ao nível de 5\%; Médias das linhas em cada sistema de cultivo seguidas por letras minúsculas iguais não diferem entre si pelo Teste de Tukey em nível de $5 \%$.

milho podem ser manejadas através da rotação de culturas, minimizando seus efeitos sobre a cultura atual.

No rendimento de grãos, foi observado que não ocorreu diferença significativa para os valores médios das duas safras avaliadas, independente do sistema de cultivo. Ao comparar o rendimento dos híbridos nas duas safras, no primeiro ano, ocorreu diferença significativa para o rendimento de grãos em ambos os sistemas de cultivo. Nesta safra, a produção do híbrido $\mathrm{P} 30 \mathrm{~F} 36$ foi até $31,8 \%$ e até $46,3 \%$ superior ao rendimento encontrado para os demais híbridos, para o sistema com rotação de culturas e monocultura, respectivamente. Esses resultados, mesmo sendo satisfatórios, devido à situação de menor volume de chuvas, quando comparados ao rendimento médio dos três híbridos obtidos na safra 2009/2010 foram 55,7 e 56,8\% inferiores para o sistema rotação e monocultura, respectivamente. Estas diferenças se devem à redução do tamanho das espigas, falhas na granação e menor peso dos grãos. Na safra 2008/2009, o PMS médio dos três híbridos foi 17,8\% e 20,7\% inferiores à safra 2009/2010, para o sistema de rotação de culturas e sistema de monocultura, respectivamente. Para essa variável também foram observadas correlações positivas entre doses de $\mathrm{N}$ nos dois sistemas de cultivo, com incrementos no PMS até a dose equivalente a $210 \mathrm{~kg}$ de $\mathrm{N} \mathrm{ha}^{-1}$.

Os valores de percentagem de GA estão apresentados na tabela 1 , que mostrou diferença significativa entre híbridos nos dois sistemas de cultivo e nas duas safras avaliadas. Na safra 2008/2009, quando ocorreu menor precipitação pluviométrica, as percentagens de GA no milho foram maiores do que as encontradas para a safra 2009/2010, chegando a 64,8\% 


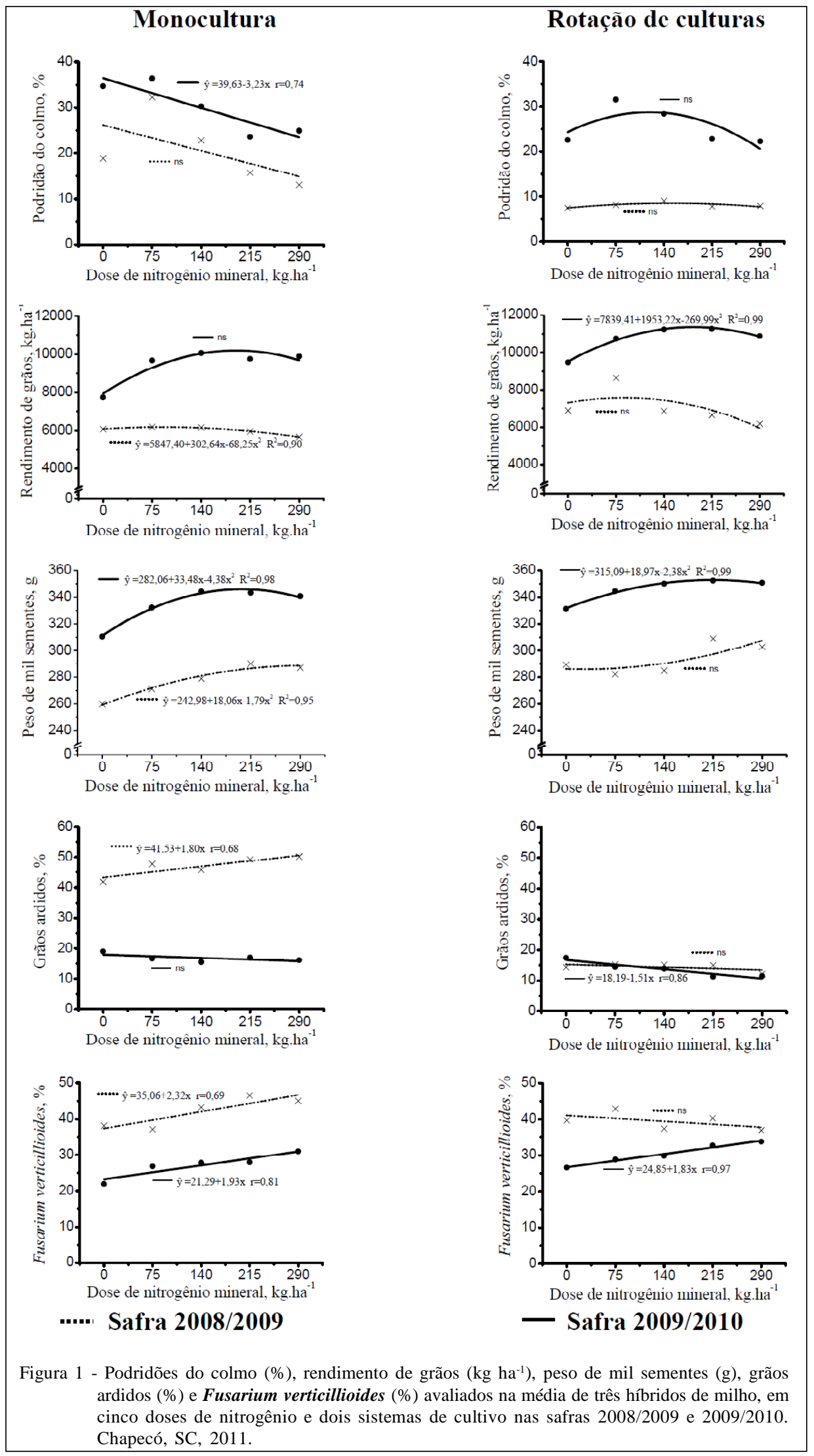

Ciência Rural, v.43, n.2, fev, 2013. 
na média dos três híbridos na área de monocultura. A alta percentagem de GA na safra 2008/2009 em monocultura pode estar relacionada à menor precipitação pluviométrica registrada no período, pois coincidiu com o enchimento de grãos e colheita. De acordo com COSTA et al. (2010), que estudaram 49 híbridos em quatro locais, a resistência genética e os regimes de chuvas são os principais fatores associados à GA na cultura do milho. Assim, em locais com clima favorável à ocorrência de GA, a escolha da cultivar é um fator de importância para o manejo da doença. Entre os híbridos testados, a ordem de grandeza para GA foi à seguinte: Maximus $>$ AS1575>P30F36, com mesma tendência para os dois sistemas de cultivo nas duas safras avaliadas. A variação média de GA entre os sistemas de cultivo e híbridos chegou a $171,1 \%$, ou seja, bem superior em monocultura.

Os fungos identificados em grãos de milho foram: D. maydis, D. macrospora, Cephalosporium sp. e $\boldsymbol{F}$. graminearum com incidência inferior a $10 \%$ das amostras analisadas, sendo que o fungo $\boldsymbol{F}$. verticillioides ocorreu em $100 \%$ das amostras. A intensidade de ataque por $\boldsymbol{F}$. verticillioides na cultura do milho pode ser observada na tabela 1 . Na média das safras avaliadas, o híbrido AS 1575 apresentou maior incidência, com mais de $41 \%$ de infecção para ambos os sistemas de cultivo. Em situação de menor precipitação pluviométrica, os valores médios para Fusarium são semelhantes nos dois sistemas de cultivo (41,7\% para monocultura e 39,0\% para rotação), porém com pequena tendência de ser maior em monocultura. Em situação normal de cultivo, sem o estresse hídrico, os valores médios são pouco maiores para rotação de culturas (29,6\% para rotação e $26,8 \%$ para monocultura). Entre híbridos, as diferenças seguiram sempre a mesma tendência, onde o AS 1575 foi o que apresentou maior suscetibilidade ao Fusarium, seguido pelo Maximus e pelo P30F36. A incidência de $\boldsymbol{F}$. verticillioides em grãos de milho, na média dos dois sistemas de cultivo, foi de $12,16 \%$ maior na primeira safra, quando ocorreu menor precipitação pluviométrica. Para essa variável, também foram observadas correlações positivas entre a quantidade de $\mathrm{N}$ aplicado e a intensidade de infecção de $\boldsymbol{F}$. verticillioides.

O fungo $\boldsymbol{F}$. verticillioides é a principal espécie produtora de fumonisinas, grupo de micotoxinas que ocorrem no milho e seus derivados (WORDELLFILHO, 2010). ROCHA (2010), que estudou a distribuição de fungos e micotoxinas em grãos de milho recém-colhidos, provenientes dos estados de São Paulo, Mato Grosso, Rio Grande do Sul e Bahia, constatou que o fungo $\boldsymbol{F}$. verticillioides foi o patógeno mais encontrado e as fumonisinas foram as micotoxinas mais frequientes nos grãos desse cereal. Entretanto, os níveis de incidência desse patógeno não foram suficientes para proporcionar diferenças significativas na presença das micotoxinas. Para os níveis de fumonisina, não foram observadas diferenças estatísticas entre os sistemas de cultivo, híbridos e doses de nitrogênio, embora a média da incidência do fungo nos grãos alcançou 34,3\%, indicando que outros fatores estão associados à incidência dessa toxina (FINK-GREMMELS, 1999) neste estudo.

A ocorrência de $\boldsymbol{F}$. verticillioides em grãos de milho e a possibilidade de produção de fumonisinas (ORSI et al., 2000) justificam a necessidade de estudos complementares, direcionados ao ponto crítico de controle na produção dessas toxinas, nos fatores climáticos envolvidos, meio de transporte, bem como nos processos empregados na secagem, os quais podem causar mudança nas condições dos grãos, bloqueando os mecanismos naturais de defesa da planta, que desencadeiam a indução de enzimas envolvidas no metabolismo secundário dessa micotoxina (KADOZAWA et al., 2006; MOREY et al., 2009). Outro aspecto a ser estudado diz respeito à diferença entre isolados do fungo para com a produção dessa toxina.

\section{CONCLUSÃO}

As podridões da base do colmo têm menor incidência em lavouras conduzidas pelo sistema de rotação de culturas, enquanto que a porcentagem de grãos com fumonisina independe do sistema de cultivo.

Os cultivos conduzidos em rotação de culturas apresentam maior rendimento por área e peso de mil sementes, com incidência de grãos ardidos inferior àqueles conduzidos em monocultura.

\section{REFERÊNCIAS}

BRASIL. Portaria n.11 de 12 de abril de 1996. Estabelece critérios complementares para classificação do milho. Diário Oficial da União, Brasília, n.72, 1996.

CASA, R.T. et al. Fungos associados à semente de milho produzida nas Regiões Sul e Sudeste do Brasil. Fitopatologia Brasileira, Brasília, v.23, n.3, p.370-373, 1998.

CASA, R.T. et al. Decomposição dos restos culturais do milho e sobrevivência saprofítica de Stenocarpella maydis e $\boldsymbol{S}$. macrospora. Fitopatologia Brasileira, Fortaleza, v.28, n.3, p.355-361. 2003. Disponível em: <http://www.scielo.br/pdf/ fb/v28n4/17007.pdf>. Acesso em: 05 jan. 2011. doi: 10.1590/ S0100-41582003000400002.

CEPA. Síntese Anual da Agricultura de Santa Catarina 2009/2010. Disponível em: <http://cepa.epagri.sc.gov.br/ 
Publicacoes/Sintese_2010/sintese\%202010_inteira.pdf>. Acesso em: 04 jan. 2011.

COMISSÃO DE QUÍMICA E FERTILIDADE DO SOLO (CQFS) RS/SC. Manual de adubação e calagem para os estados do Rio Grande do Sul e Santa Catarina. Porto Alegre: Sociedade Brasileira de Ciência do Solo - Núcleo Regional Sul, 2004. 400p.

COSTA, R.V. et al. Recomendações de cultivares de milho para a resistência a grãos ardidos. Sete Lagoas: EmbrapaCNPMS, 2010. 8p. (EMBRAPA-CNPMS, Circular Técnica, 154).

DEACON, J.W. Modern mycology. 3.ed. Oxford: Blackwell Science 1997. 312p.

DENTI, E.A.; REIS, E.M. Efeito da rotação de culturas, da monocultura e da densidade de plantas na incidência das podridões da base do colmo e no rendimento de grãos do milho. Fitopatologia Brasileira, Brasília, v.26, p.635-639, 2001. Disponível em: <http://www.scielo.br/pdf/fb/v26n3/a09v26 n3.pdf>. Acesso em: 03 ago. 2011.

FANCELLI, A.L. Milho e feijão: elementos para manejo em agricultura irrigada. Piracicaba: Departamento de agricultura/ ESALQ/USP, 1994. 14p.

FARIAS, A.X. et al. Fumonisinas em milho: um risco à saúde humana e animal. Rio de Janeiro: Embrapa-CTAA, 2002. 2p. (EMBRAPA-CTAA, Comunicado Técnico, 51).

FINK-GREMMELS, J.H. Micotoxins: their implications for human and animal health. Veterinary Quarterly, The Hague, v.21, n.4, p.115-120, 1999.

FOLEY, D.C.; WERNHAM, C.C. The effect of fertilizers on stalk rot of corn in Pennsylvania. Phytopathology, East Lansing.47, p.11-12, 1957.

FONSECA, H. SISTEMA DE Amostragem para análise de aflatoxinas em grãos. Revista Microbiologia, São Paulo, v.21, n.2, p.66-70, 1991.

KADOZAWA, P. et al. Atividade inibidora de amilase e protease de milho contra Fusarium verticillioides durante a germinação. Semina: Ciências Agrárias, Londrina, v.27, p.231-242, 2006.

MOREY, A.T. ET AL. AVALiação de fontes de carbono e nitrogênio na produção de fumonisina B1 por Fusarium verticillioides em meio líquido definido. Semina: Ciências Agrárias, Londrina, v.30, p.647-654, 2009.

ORSI, R.B. et al. Mycoflora and occurrence of fumonisins in freshly harvested and stored hybrid maize. Journal Stored Products Research, Oxford, v.36, n.1, p.75-87, 2000.

PINTO, N.F.J.A. Patologia de sementes de milho. Sete Lagoas: Embrapa-CNPMS, 1998. 44p. (EMBRAPA-CNPMS, Circular Técnica, 29).

PORTAL PIONEER - HIBRIDOS DE MILHO - 30F36. DISPONÍVEL em: <http://www.pioneersementes.com.br/>. Online. Acesso em: 05 abr. 2008.

REIS, E.M. et al. Método para quantificar os danos no rendimento de grãos causados pelas podridões da base do colmo do milho. Fitopatologia Brasileira, Brasília, v.23, p.300. 1998.

ROCHA, L.O. Distribuição de fungos e micotoxinas em grãos de milho recém-colhidos e variabilidade genética das cepas de Fusarium verticillioides e Aspergillus flavus isoladas. 2010. 174f. Tese (Doutorado em Microbiologia) Instituto de Ciências Biomédicas, Universidade de São Paulo, São Paulo, SP.

SHURTLEFF, M.C. Compendium of corn diseases. St. Paul: American Phytopathological Society, 1992. 105p.

WORDELL FILHO, J.A.; CASA, R.T. Doenças na cultura do milho. In: WORDELL FILHO, J.A.; ELIAS, H.T. (Eds.). A cultura do milho em Santa Catarina. Florianópolis: Epagri, 2010. p.207-272.

WORDELL FILHO, J.A. Micotoxinas na cultura do milho. Agropecuária Catarinense, Florianópolis, v.23, p.46-48, 2010.

WORDELL FILHO, J.A. et al. Podridões da base do colmo na cultura do milho: sintomas e medidas de controle. Agropecuária Catarinense, Florianópolis, v.21, p.47-49, 2008.

ZONTA, E.P.; MACHADO, A.A. SANEST - Sistema de análise estatística para microcomputadores. Pelotas: DMEC/IFM/UFPel, 1987. 138p.

ZUBER, M.S. et al. Studies on the interrelation of tild stalk lading, two stalk rotting fungi, and chemical composition of corn. Agronomy Journal, v.49, p.328-331, 1957. 